\title{
Advances in Extreme-scale 3D EM: Specimen Preparation and Recording Systems for Electron Microscopic Tomography and Serial Block Face SEM.
}

\author{
Mark H. Ellisman, Thomas J. Deerinck, Eric Bushong, James C. Bouwer, Tristan Shone, Liang Jin, \\ Anna Milazzo, Steven Peltier, and Nguyen-Hu Xuong. \\ University of California, San Diego, National Center for Microscopy and Imaging Research, 9500 \\ Gilman Dr., BSB 1000, La Jolla, Ca., USA 92093-0608
}

\begin{abstract}
:
Introduction: A host of new technological tools for data acquisition and processing are beginning to deliver new syntheses of information about biological systems, including new revelations regarding the complexities of the brain. Brain researchers are now beginning to be able to explore across the full range of scales, from genomics and molecular structure to networks of neuronal systems. Gaps in knowledge and limited abilities to span scales in tissues highlight the need for tools and methods that will allow the acquisition of high fidelity 3D image information at high resolution, but over very large expanses. Thus, despite rapid progress in development of new experimental methods, our ability to simultaneously study the brain across many key scales remains quite limited. To state the problem simply and specifically: experimental methodologies available today reveal only limited views of nervous system organization at the tissue and subcellular-supramolecular levels. Consequently many research groups are now working to expand data acquisition and computational methods to address the grand challenge of understanding how the structure of the brain gives rise to complex functions. Our group is deeply involved in this activity and has developed many tools that accelerate the delivery of large and detailed views of elements of the nervous system, extending to the nanometer scale. Several of our ongoing projects related to these multiscale challenges will be highlighted in this presentation. These include work on advanced extreme-scale and high quality image recording systems for electron tomography; development of specimens and procedures to increase the image quality, resolution and field of view for 3D volumes acquired by serial block face scanning electron microscopy.
\end{abstract}

Advanced Detection Systems for TEM: Film has long been regarded as the gold standard for image recording. When scanning to achieve a (modest) dynamic range of 3 orders of magnitude, a true format of approximately $8 \mathrm{k} \times 6.5 \mathrm{k}$ can be achieved. This still far exceeds the performance of current digital detection systems for TEM, though is well short of theoretical resolution based on grains size alone owing to the need to expose many grains to achieve wide dynamic range. While film provides excellent modulation transfer function (MTF), especially as compared with commercially available CCD cameras, it requires several post-acquisition steps like development and digitizing that are cumbersome and time-consuming. We have developed and tested a lenscoupled system that matches the resolution and format of film with a far superior dynamic range. Our goal was to build a large format high-resolution wide-field imaging detector with single electron sensitivity. Key to this goal is recording unique information in every pixel of the image by ensuring modulation transfer of at least $10 \%$ to the Nyquist limit.

We have built an $8 \mathrm{k} \times 8 \mathrm{k}$ lens-coupled camera system, where the electron image produced by the microscope is first converted to a photon image with the use of a patented, large and modular self-supporting P43 phosphor scintillation screen. A set of four custom-designed high numerical aperture, demagnification lenses surrounding the central pyramidal beam splitter collect the light and 
relay the quadrant images to four individually housed, custom designed $4 \mathrm{k} \times 4 \mathrm{k}$, high dynamic range, back-thinned, back-side illuminated CCD sensors with a pixel pitch of $15 \mu \mathrm{m}$. The fully integrated camera system achieves single-electron sensitivity while delivering useable MTF right to Nyquist. The large format, high-resolution, and sensitivity of this digital imaging system, together with the linearity and large dynamic range of the CCDs, make this $8 \mathrm{k} \times$ 8k device a suitable replacement for film - with superior performance in many aspects. We are now using this system to acquire electron tomographic data from serial sections of biological material, including mosaics of multiple $8 \mathrm{k} \mathrm{x} 8 \mathrm{k}$ frames, automatically at $400 \mathrm{keV}$. We will demonstrate the application of this extreme scale camera system to reconstruct entire cells of the nervous system by serial section electron tomography.

Since it is the scintillator and the accompanying fiber optic that limit resolution in commercial CCD-based digital imaging devices for TEM, we set out (approximately 10 years ago) to develop scintillator-less imaging devices composed of radiation hardened CMOS image sensors that could detect and record intermediate voltage electron events directly in silicon. These detectors have been named Direct Detection Devices $\left(D_{D D}^{\circledR}\right)$. We have tested 7 generations of DDDs at accelerating voltage from $120 \mathrm{keV}$ to $400 \mathrm{keV}$. From the $4^{\text {th }}$ generation onward, the DDDs have been put into production situations and used for tomography, low-dose imaging, and wide-field mosaic acquisition. The latest generation of DDD is $4 \mathrm{k} \times 3 \mathrm{k}$ device featuring 6-micron pixels and a 30:1 signal to noise ratio at $300 \mathrm{keV}$ with a readout rate of 480 million pixels per second. Examples of the application of the DDD to biological imaging projects, including automated electron tomography acquisitions will be shown.

Serial Block Face Imaging with an SEM: Ultrascale high resolution volumes of cells and tissues also may be obtained by repeated SEM imaging of plastic-embedded material after removal of the surface layer by either an ultramicrotome or ion milling (FIBSEM). We chose to focus our effort on enhancing the utility of the ultramicrotome-based approach rather than FIBSEM as the field of view possible with this method, (approximately $1 \mathrm{~mm}$ today), significantly greater than that which can be obtained with particle beam milling methods. Furthermore, the stability of the specimen and continuous ability to remove equally thin planes of material is practical now with the SBFSEM approach, whereas there are challenges in this regard with the FIBSEM approach. To make SBFSEM more useful we first needed to improve the method of introducing heavy metal contrast into the specimen so we developed new staining protocols. These provide for much greater specimen contrast and also enhance the conductivity of the specimen blocks, which help reducing specimen charging. By careful assessment of the excitation volume we have also developed a practical strategy to match the information retrieved from each scan to the thickness of material removed from each pass of the in column ultramicrotome (we are using a Gatan 3View). Results of work on specimens contrasted with different methods and over large extents at modest resolution and more limited extents at high resolution with an FEI Quanta FEG will be shown. In addition, the near-term prospects for extending the field of view, sensitivity and axial resolution of this method will be discussed. 